\title{
Respiratory syncytial virus: co-infection and paediatric lower respiratory tract infections
}

\author{
Lay-Myint Yoshida ${ }^{1,7}$, Motoi Suzuki ${ }^{1,7}$, Hien Anh Nguyen², Minh Nhat Le ${ }^{1,2}$, \\ Thiem Dinh Vu², Hiroshi Yoshino ${ }^{1}$, Wolf-Peter Schmidt ${ }^{1,3}$, Thi Thuy Ai Nguyen ${ }^{4}$, \\ Huu Tho Le ${ }^{5}$, Konosuke Morimoto', Hiroyuki Moriuchi', Duc Anh Dang ${ }^{2}$ and \\ Koya Ariyoshi ${ }^{1}$
}

Affiliations: 'Dept of Clinical Medicine, Institute of Tropical Medicine, Nagasaki University, Nagasaki, and ${ }^{6}$ Nagasaki University Hospital, Nagasaki University, Nagasaki, Japan. ${ }^{2}$ National Institute of Hygiene and Epidemiology, Hanoi, ${ }^{4}$ Khanh Hoa General Hospital, Nha Trang, and ${ }^{5}$ Khanh Hoa Health Service, Nha Trang, Vietnam. ${ }^{3}$ London School of Tropical Medicine and Hygiene, London, UK. ${ }^{7}$ Both authors contributed equally.

Correspondence: K. Ariyoshi, Dept of Clinical Medicine, Institute of Tropical Medicine, Nagasaki University 1-12-4, Sakamoto, Nagasaki, 852-8523, Japan. E-mail: karidanagasaki-u.ac.jp

ABSTRACT Comprehensive population-based data on the role of respiratory viruses in the development of lower respiratory tract infections (LRTIs) remain unclear. We investigated the incidence and effect of single and multiple infections with respiratory viruses on the risk of LRTIs in Vietnam.

Population-based prospective surveillance and a case-control study of hospitalised paediatric patients with acute respiratory infection (ARI) were conducted from April 2007 through to March 2010. Healthy controls were randomly recruited from the same community. Nasopharyngeal samples were collected and tested for 13 respiratory viruses using multiplex PCRs.

1992 hospitalised ARI episodes, including 397 (19.9\%) with LRTIs, were enrolled. Incidence of hospitalised LRTIs among children aged $<24$ months was 2171.9 per 100000 (95\% CI 1947.9-2419.7). The majority of ARI cases $(60.9 \%)$ were positive for at least one virus. Human rhinovirus $(24.2 \%)$, respiratory syncytial virus (20.1\%) and influenza A virus (12.0\%) were the most common and $9.5 \%$ had multiple-viral infections. Respiratory syncytial virus and human metapneumovirus infections independently increased the risk of LRTIs. Respiratory syncytial virus further increased the risk, when co-infected with human rhinovirus, human metapneumovirus and parainfluenza virus-3 but not with influenza A virus. The casecontrol analysis revealed that respiratory syncytial virus and influenza A virus increased the risk of ARI hospitalisation but not human rhinovirus.

Respiratory syncytial virus is the leading pathogen associated with risk of ARI hospitalisation and LRTIs in Vietnam.

-

@ERSpublications

RSV is the leading pathogen associated with risk of ARI hospitalisation and LRTIs in Vietnam http://ow.ly/lvdXs

This article has supplementary material available from www.erj.ersjournals.com

Received: July 032012 | Accepted after revision: Sept 212012 | First published online: May 032013

Support statement: This study was funded by the Japan Initiative for Global Research Network on Infectious Diseases (JGRID), the Ministry of Education, Culture, Sports, Science and technology (MEXT, Tokyo, Japan) and Japan Society for Promotion of Science (JSPS), Japan. Wolf-Peter Schmidt was funded by JSPS. The funding agencies had no role in designing, conducting, analysing data or preparing the manuscript.

Conflict of interest: None declared.

Copyright @ERS 2013 


\section{Introduction}

Acute respiratory infection (ARI) is the leading cause of mortality and morbidity among children worldwide $[1,2]$. Studies have established that Streptococcus pneumoniae and Haemophilus influenzae type b are the major bacterial causes of childhood pneumonia in developed and developing countries [3, 4]. Viruses also play critical roles in the development of ARI or pneumonia in young children. However, elucidating the roles of viral pathogens may be complex as a wide range of viruses, such as respiratory syncytial virus (RSV), human rhinovirus (HRV), influenza virus (FLU) A and FLU B, parainfluenza virus (PIV) and human metapneumovirus (hMPV), are associated with severe forms of childhood ARI including bronchiolitis and pneumonia [5-9]. A systematic review has estimated that $22 \%$ of severe ARI episodes among children are related to RSV infections [10]. Still, hard evidence or population-based data on the burden of respiratory viruses on paediatric ARIs in developing countries, especially in Southeast Asia has been lacking.

We have previously reported that HRV, RSV and FLU A were the most commonly detected viral agents among hospitalised ARI patients based on our 14-month study in central Vietnam [11]. However, yearly differences in viral outbreaks may influence the annual incidence of hospitalised ARI or pneumonia cases. Data from healthy children are also important since respiratory viruses, like HRV, have been detected in 5$18 \%$ of asymptomatic children and its role on paediatric ARI is still controversial $[6,12]$.

Recent progress in molecular microbiology techniques, such as multiplex PCR, has made it possible to simultaneously detect multiple viruses with high sensitivity and specificity [13], and has revealed that multiple viral infections in children with ARIs are not uncommon [7, 14]. However, the contribution of respiratory viruses to severe ARI either as single or multiple infections needs further clarification. In particular, the combination of respiratory viruses that increase the risk of childhood lower respiratory tract infections (LRTIs) has not been thoroughly investigated.

Thus, we conducted a population-based prospective surveillance and a case-control study covering multiple years to precisely elucidate the incidence and effect of single and multiple respiratory viral infections on paediatric ARI hospitalisation and risk of LRTIs in central Vietnam.

\section{Methods}

\section{Study site and study population}

The surveillance was conducted at Khanh Hoa General Hospital (Nha Trang, Vietnam), which is located in the centre of Nha Trang and is the only hospital in the catchment area that provides primary, secondary and tertiary care for the population. After the implementation of "the law on child care, protection and education" in 2005, all Vietnamese children $<6$ years of age have been eligible to receive free medical care, which includes treatment and regular check-ups in the public sector. Thus, we can reasonably expect that almost all children aged $<5$ years with severe ARI who required hospitalisation would have been admitted to Khanh Hoa General Hospital.

A population-based cross-sectional survey was conducted from June 8, 2006 to July 25, 2006 covering all residents of the catchment area. Overall, 198729 individuals living in 42770 households from 16 communities in Nha Trang were enrolled. Among them, 13935 were children aged $<5$ years. Detailed methods and characteristics of the study populations have been described previously [15]. This census population formed the source population for our case-control study. In addition, this population was treated as a cohort for the calculation of incidence rates. The coverage of an extended programme of immunisation reaches $95 \%$ in this area; however, pneumococcal conjugate vaccine and $H$. influenzae type B conjugate vaccine had not been introduced at the time of this study.

\section{Hospital case enrolment and data collection}

Children admitted to Khanh Hoa General Hospital from April 1, 2007 to March 31, 2010 who: 1) presented with cough and/or difficulty breathing, 2) were aged between 1 month and 60 months upon admission, and 3) resided in our catchment area (16 communities in Nha Trang), were enrolled in the study. On admission, after obtaining informed consent from the parents or guardians, clinical-epidemiological information was collected by paediatricians in the Khanh Hoa General Hospital. Nasopharyngeal and blood samples were taken within $24 \mathrm{~h}$ of admission by trained research nurses. To achieve good quality data, all processes from the enrolment to data and sample collection were monitored by a research doctor and a research nurse. The information on disease onset has been collected since January 2009.

\section{Case definitions of LRTIs}

Case categories were defined using modified World Health Organization (WHO) Integrated Management of Childhood Illnesses (IMCI) algorithms [16]. Children with general danger signs, chest indrawing or stridor were categorised as severe LRTIs. The presence of severe LRTIs or tachypnoea (a respiratory rate 
$>60 \mathrm{~min}^{-1}$ for children aged $1 \mathrm{month},>50 \mathrm{~min}^{-1}$ for those aged 2-11 months and $>40 \mathrm{~min}^{-1}$ for those aged 12-59 months) were categorised as LRTIs.

\section{Enrolment of healthy controls}

350 healthy children aged $<5$ years were randomly selected from two communities in the study area, based on the census data. Eligible children were free of fever, signs and symptoms of ARI, and had no history of antibiotic use within 1 month of the evaluation. In July 2008, children were invited to community health centres where a paediatrician performed a clinical examination to confirm their healthy status. After obtaining informed consent, nasopharyngeal swab samples were collected in the same way as they were for children hospitalised for ARI.

\section{Laboratory analysis}

Nasopharyngeal samples collected at the time of admission were stored at $-80^{\circ} \mathrm{C}$. Nucleic acid extraction was carried out using the QIAamp viral RNA extraction kit (Qiagen, Tokyo, Japan). Four, inhouse, multiplex PCR assays: 1) FLU A, FLU B, RSV, hMPV; 2) PIV 1, 2, 3, and 4; 3) HRV, human coronavirus 229E (HCoV-229E), human coronavirus OC43 (HCoV-OC43); and 4) adenovirus (AdV) and human bocavirus ( $\mathrm{HBoV}$ ) were performed, using RT-PCR reagents (Qiagon), to detect 13 respiratory viruses in each sample, as described previously [11].

\section{Data management and analysis}

All data were double-entered and validated by trained data management staff at the Khanh Hoa provincial health service using FoxPro 9.0 (Microsoft, Redmond, WA, USA) database. The incidences of hospitalised ARIs, hospitalised viral respiratory infections, and hospitalised LRTIs among the study population were calculated. The 95\% confidence intervals for incidences were calculated using Wilson score method. Clinical characteristics were compared between LRTI categories using Chi-squared test for trend and ANOVA.

To evaluate the effect of respiratory viral infection on the risk of ARI admissions, case-control analysis was performed. The dataset comprised ARI patients hospitalised from June 2008 to August 2008 and 350 healthy control subjects enrolled in July 2008. Odds ratios were calculated using logistic regression models.

Respiratory viruses single or multiple infections, and risk of LRTIs among hospitalised children were also studied. The effect of each viral infection on the risk of an ARI being diagnosed as a LRTI was assessed using Poisson regression [17]. Confidence intervals for risk ratios were adjusted for individual-level correlation using robust standard errors. Multivariate analysis was performed to adjust for the effects of other viruses. Finally, to evaluate the risk ratios of single- and multiple-infection, Mantel's score tests for trend were performed adjusting for other viruses. STATA 10 (Stata corp., College Station, TX, USA) was used for all statistical analyses.

\section{Ethics}

The study was approved by institutional review boards in the National Institute of Hygiene and Epidemiology, Vietnam and the Institute of Tropical Medicine, Nagasaki University, Japan.

\section{Results}

\section{Characteristics of hospitalised ARI cases}

During the study period, a total of 2035 cases from the catchment area were admitted and 1786 children with 1992 hospitalised ARI episodes (97.9\%) who gave informed consent were enrolled in the study. Among them, 1622 children had one episode, while 264 had two episodes and 106 had three or more ARI episodes. Characteristics of the hospitalised ARI cases are shown in table 1. Among 1992 ARI cases, 397 (19.9\%) were categorised as LRTIs including 113 cases of severe LRTIs (5.7\% of all episodes). The mean duration from disease onset to admission was $2.9 \pm 3.5$ days $(n=753)$. Younger age, lower body temperature, higher pulse rate, presence of wheeze and malnutrition were associated with higher clinical severity or LRTIs. Children with severe LRTIs were hospitalised for longer than those without severe LRTIs and received steroid therapy more frequently. The use of antibiotics before admission tended to be less among severe LRTIs.

\section{Incidences of hospitalised paediatric ARI, LRIs and viral infections}

The annual incidences of hospitalised episodes of ARI and LRTIs among children aged $<5$ years were 4765 and 949.6 per 100000 child-years, respectively. Higher incidences were observed among children aged $<24$ months than among those aged $24-60$ months. There was no significant difference in the incidences between children aged $<12$ months and those aged $<24$ months (table 2).

Among the 1992 episodes, 1214 (60.9\%) were positive for any of the 13 viruses tested. HRV (481, 24\%), RSV $(401,20 \%)$ and FLU A $(239,12 \%)$ were the most common viruses detected, followed by AdV (111, 6\%), PIV3 
TABLE 1 Demographic and clinical features of hospitalised acute respiratory infection cases, grouped by clinical severity, in the Khanh Hoa Province in Vietnam from 2007 to 2010

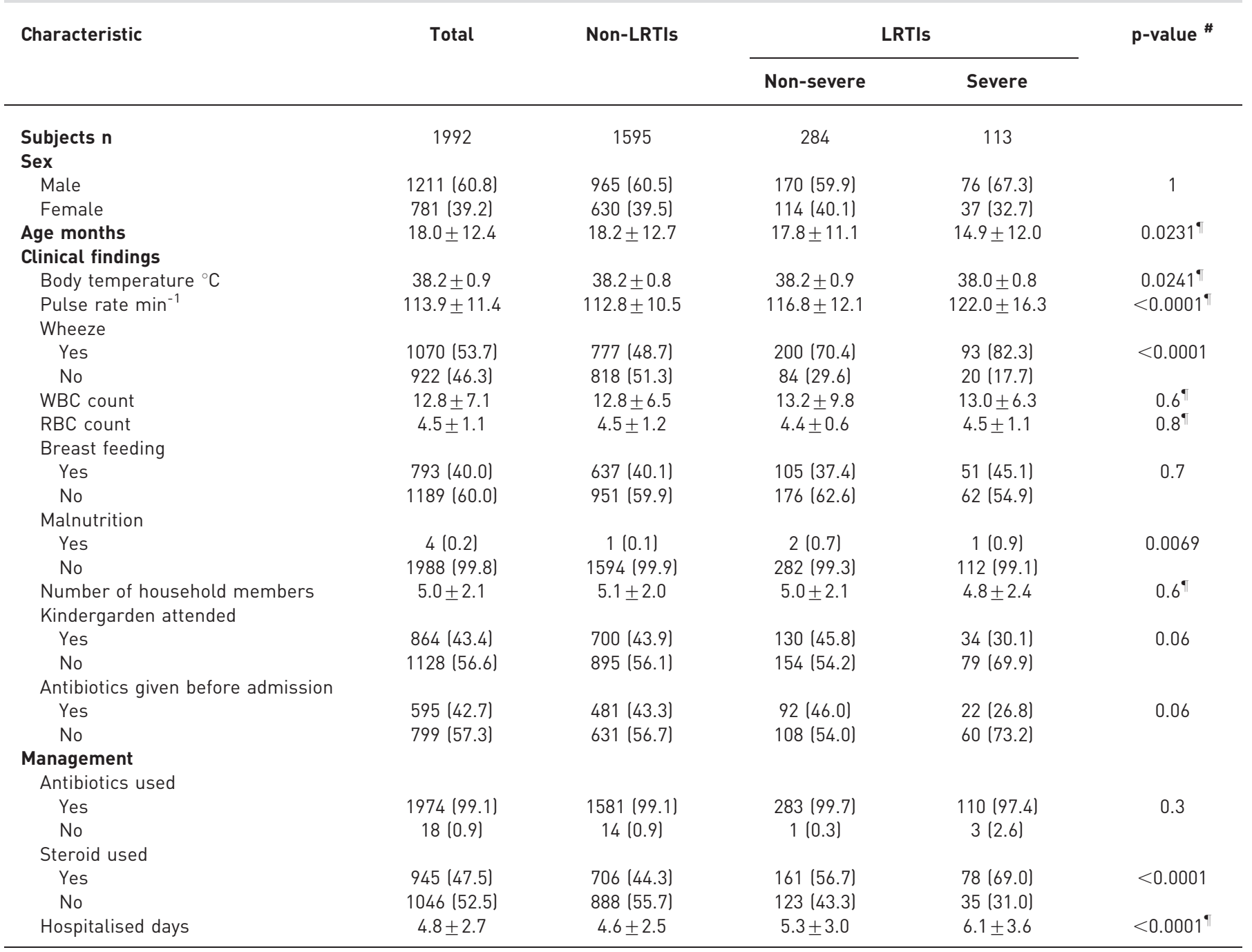

Data are presented as $\mathrm{n}(\%)$ or mean \pm SD, unless otherwise stated. LRTIs: lower respiratory infections; WBC: white blood cell; RBC: red blood cell. \#: Chi-squared test for trend unless otherwise indicated; ": ANOVA.

(49, 2.4\%), hMPV $(44,2 \%)$ and $\operatorname{HBoV}(40,2 \%)$. Co-infections with two or more respiratory viruses were common. Of all ARI episodes, 189 (9.5\%) were multiple viral infections which included 174 (8.7\%) dual infections and $15(0.8 \%)$ triple infections (online supplementary figure S1). Among the 15 triple infections, 10 included RSV: five were combinations of RSV, HRV and FLU A; two were RSV, HRV and AdV; another two were RSV, FLU A and AdV; and one was RSV, FLU A and hMPV. High incidences of paediatric hospitalised ARI cases associated with HRV, RSV and FLU A infection were observed among all age groups of children in Nha Trang (table 2). The incidence rates of hMPV and FLU B differed considerably year by year, but the other viruses did not (online supplementary figure S2).

In terms of seasonality our data, covering three consecutive years including the peak of H1N1 swine flu in October 2009, showed that every year there is a clear seasonal pattern for RSV infection during the summer season (from June to November), whereas other viruses including HRV and FLU A did not show any discrete seasonality (online supplementary fig. S2).

Virus detection in apparently healthy children and the risk of ARI hospitalisation Subclinical HRV infection was reported to be common since it has been detected in apparently healthy children $[6,12]$. In order to investigate the pathological role of respiratory viruses, including HRV among children, we compared the virus detection rates between the ARI inpatients and 350 healthy children. Among the healthy controls enrolled in July 2008, 147 (42\%) were positive for any of the tested viruses: 
TABLE 2 Annual incidence (per 100 000) of hospitalisations with acute respiratory infection (ARI), viral respiratory infections and lower respiratory tract infections (LRTIs) among children aged $<5$ years in the Khanh Hoa Province of Vietnam

\begin{tabular}{lcc} 
& Children aged $<\mathbf{2 4}$ months & Children aged $<60$ months \\
\hline Hospitalised ARI & $10282.7(9847.1-10728.8)$ & $4765(4574.5-4962)$ \\
LRTIs & $2171.9(1947.9-2419.7)$ & $949.6(861.8-1046.1)$ \\
Severe LRTIs & $626.6(509.5-770.1)$ & $270.3(225-324.7)$ \\
HRV & $2663.3(2415.7-2933.8)$ & $1150.6(1053.7-1256)$ \\
RSV & $2342.8(2110.3-2598.8)$ & $959.2(870.9-1056.2)$ \\
FLU A & $1039.7(886.1-1218.9)$ & $571.7(504.2-648.1)$ \\
Adenovirus & $662.3(541.5-809.2)$ & $265.5(220.6-319.4)$ \\
PIV3 & $284.8(209.4-387.2)$ & $117.2(88.7-154.8)$ \\
hMPV & $220.8(155.7-312.8)$ & $105.3(78.4-141.2)$ \\
HBoV & $242.1(173.4-337.7)$ & $95.7(70.3-130.2)$ \\
FLU B & $99.7(59.4-167.1)$ & $52.6(34.8-79.6)$ \\
PIV1 & $71.2(38.7-130.9)$ & $43.1(27.2-68)$ \\
PIV2 & $28.5(11.1-73.2)$ & $26.3(14.7-47.1)$ \\
Coronavirus & $0(0-0)$ & $4.8(1.3-17.4)$ \\
PIV4 & $7.1(1.3-40.3)$ & $4.8(1.3-17.4)$ \\
\hline
\end{tabular}

Data are presented as n (95\% CI). HRV: human rhinovirus; RSV: respiratory syncytial virus; FLU: influenza virus; PIV: parainfluenza virus; hMPV: human metapneumovirus; HBoV: human bocavirus.

HRV (35\%) was by far the most commonly detected, followed by AdV (4.6\%), RSV (2.9\%) and FLU A (1\%). Comparison with children hospitalised with ARI during the same period (June-August, 2008, $\mathrm{n}=148$ ) showed that RSV and FLU A, but not HRV, were associated with the increased risk of hospitalisation (table 3).

\section{Viral ARI and risk of LRTIs}

The effect of respiratory viral infection on the risk of an ARI being clinically diagnosed as an LRTI is shown in table 4. In univariate analysis, RSV and hMPV were associated with the diagnosis of LRTIs. In multivariate analysis adjusting for other viral co-infections, HRV, RSV and hMPV infections were independent risk factors for LRTIs among children. These three viruses also demonstrated an increasing trend of risk between non-severe and severe LRTIs (online supplementary table S1). Child sex, age and

TABLE 3 Comparison between hospitalised children with acute respiratory infections (ARIs) and healthy controls in the Khanh Hoa Province of Vietnam in 2008

\section{Case}

\begin{tabular}{lccc}
\hline Subjects & 148 & 350 & \\
Any Virus & $100(67.6)$ & $147(42)$ & $2.88(1.92-4.31)$ \\
HRV & $43(29.1)$ & $121(34.6)$ & $0.78(0.51-1.18)$ \\
RSV & $58(39.2)$ & $10(2.9)$ & $21.91(10.77-44.58)$ \\
FLU A & $13(8.9)$ & $4(1.1)$ & $8.33(2.67-26)$ \\
AdV & $8(5.4)$ & $16(4.6)$ & $1.19(0.5-2.85)$ \\
PIV3 & $0(0)$ & $0(0)$ & \\
hMPV & $0(0)$ & $0(0)$ & $0.59(0.07-5.31)$ \\
HBoV & $1(0.7)$ & $4(1.1)$ & $2.37(0.15-38.21)$ \\
FLU B & $1(0.7)$ & $1(0.3)$ & \\
PIV1 & $0(0)$ & $0(0)$ & \\
PIV2 & $0(0)$ & $2(0.3)$ & \\
HCoV & $0(0)$ & $0(0)$ & \\
PIV4 & $0(0)$ & $(0)$ & \\
\hline
\end{tabular}

Data are presented as $\mathrm{n}$ or $\mathrm{n}(\%)$, unless otherwise stated. Cases are children with ARI aged $<5$ years hospitalised from June to August 2008. Controls are healthy children aged $<5$ years randomly selected from two communities in the catchment area. HRV: human rhinovirus; RSV: respiratory syncytial virus; FLU: influenza virus; AdV: adenonvirus; PIV: parainfluenza virus; hMPV: human metapneumovirus; HBoV: human bocavirus; HCoV: human coronavirus. 
TABLE 4 Effects of respiratory viral infection on the risk of lower respiratory infections (LRTIs) among hospitalised children aged $<5$ years in the Khanh Hoa Province in Vietnam from 2007 to $2010^{\#}$

\begin{tabular}{|c|c|c|c|c|c|}
\hline & RT-PCR & Non-LRTIs & LRTIS & $\begin{array}{l}\text { Unadjusted risk } \\
\text { ratio }(95 \% \mathrm{CI})\end{array}$ & $\begin{array}{l}\text { Adjusted risk } \\
\text { ratio }(95 \% \mathrm{CI})\end{array}$ \\
\hline Subjects & & 1595 & 397 & & \\
\hline \multirow[t]{2}{*}{ HRV } & Positive & $371(77.1)$ & $110(22.9)$ & $1.2(0.99-1.46)$ & $1.26(1.03-1.54)$ \\
\hline & Negative & 1224 (81.0) & $287(19.0)$ & 1 & 1 \\
\hline \multirow[t]{2}{*}{ RSV } & Positive & $307(76.6)$ & $94(23.4)$ & $1.23(1.00-1.51)$ & $1.3(1.05-1.59)$ \\
\hline & Negative & $1288(81.0)$ & $303(19.0)$ & 1 & 1 \\
\hline \multirow[t]{2}{*}{ FLU A } & Positive & $203(84.9)$ & $36(15.1)$ & $0.73(0.53-1.00)$ & \\
\hline & Negative & $1392(79.4)$ & $361(20.6)$ & 1 & \\
\hline \multirow[t]{2}{*}{ AdV } & Positive & $95(85.6)$ & $16(14.4)$ & $0.71(0.44-1.16)$ & \\
\hline & Negative & $1500(79.7)$ & $381(20.3)$ & 1 & \\
\hline \multirow[t]{2}{*}{ PIV3 } & Positive & $38(77.5)$ & $11(22.5)$ & $1.13(0.67-1.92)$ & \\
\hline & Negative & $1557(80.1)$ & $386(19.9)$ & 1 & \\
\hline \multirow[t]{2}{*}{ hMPV } & Positive & $30(68.2)$ & $14(31.8)$ & $1.62(1.04-2.52)$ & $1.72(1.1-2.68)$ \\
\hline & Negative & 1565 (80.3) & $383(19.7)$ & 1 & 1 \\
\hline \multirow[t]{2}{*}{ HBoV } & Positive & 33 (82.5) & 7 (17.5) & $0.88(0.44-1.73)$ & \\
\hline & Negative & $1562(80.0)$ & $390(20.0)$ & 1 & \\
\hline \multirow[t]{2}{*}{ FLU B } & Positive & $19(86.4)$ & $3(13.6)$ & $0.68(0.24-1.95)$ & \\
\hline & Negative & $1576(80.0)$ & $394(20.0)$ & 1 & \\
\hline \multirow[t]{2}{*}{ PIV1 } & Positive & $17(94.4)$ & $1(5.56)$ & $0.28(0.04-1.87)$ & \\
\hline & Negative & 1578 (79.9) & $396(20.1)$ & 1 & \\
\hline \multirow[t]{2}{*}{ PIV2 } & Positive & 9 (81.8) & 2 (18.2) & $0.91(0.26-3.2)$ & \\
\hline & Negative & $1586(80.1)$ & $395(19.9)$ & 1 & \\
\hline
\end{tabular}

Data are presented as $\mathrm{n}$ or $\mathrm{n}(\%)$, unless otherwise stated. HRV: human rhinovirus; RSV: respiratory syncytial virus; FLU: influenza virus; AdV: adenovirus; PIV: parainfluenza virus; hMPV: human metapneumovirus; HBoV: human bocavirus. ${ }^{\#}: \mathrm{n}=1992$.

month of admission were not included in these models as confounders because they did not change the effect size of viral infections. FLU A infection was not associated with increased risk of LRTIs among hospitalised ARI patients even after adjusting for other viruses (adjusted risk ratio 0.77, 95\% CI 0.56-1.06, data not shown in table).

\section{Viral co-infection and risk of LRTIs}

To investigate the effects of respiratory virus co-infections on the development of paediatric LRTIs, we further analysed the risk of LRTIs by comparison of three groups: virus negative, single virus positive and dual virus positive. Overall, viral co-infection did not increase the risk of LRTIs (test for interaction, $\mathrm{p}>0.1$ ). However, there was good evidence (score test for trend) that dual infection with RSV and HRV, RSV and PIV3, or RSV and hMPV led to a higher risk of pneumonia than single infection with any of these viruses (fig. 1). Interestingly, FLU A plus RSV or FLU A plus co-infection with any other virus did not increase the risk for LRTIs. These associations were observed regardless of the child's age and month of admission.

\section{Discussion}

This large scale, comprehensive, population-based study, undertaken over 4 years, elucidated the burden of respiratory viruses on paediatric ARI hospitalisation and LRTIs in central Vietnam and enabled us to elucidate the impact of each viral infection or co-infection on the development of LRTIs.

\section{RSV as leading viral pathogen causing ARI hospitalisation in Nha Trang, Central Vietnam}

We quantitatively clarified the burden of each viral pathogen on paediatric ARIs and demonstrated that RSV is by far the most important viral pathogen to cause paediatric ARIs requiring hospitalisation in Vietnam, which is compatible with data from a number of other studies [5, 18, 19]. Interestingly, our three consecutive years of observation showed the peak of RSV disease always occurred in the dry hot season. This endemic pattern is distinct from that in countries in the temperate zone, where RSV outbreaks normally occur during the winter season $[9,18,20]$. We found that influenza virus was the second most important virus causing paediatric ARI hospitalisations and circulated throughout the year in this area without a clear peak. Information on the seasonality of respiratory viruses is very important for future preventive strategies against viral ARIs in Vietnam. 
a)

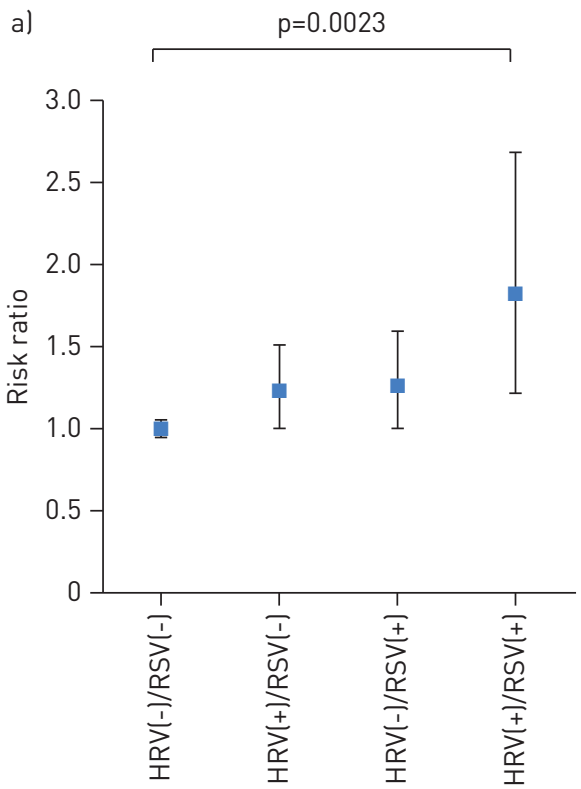

d)

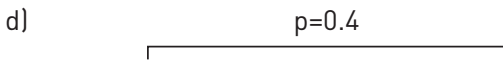

b)

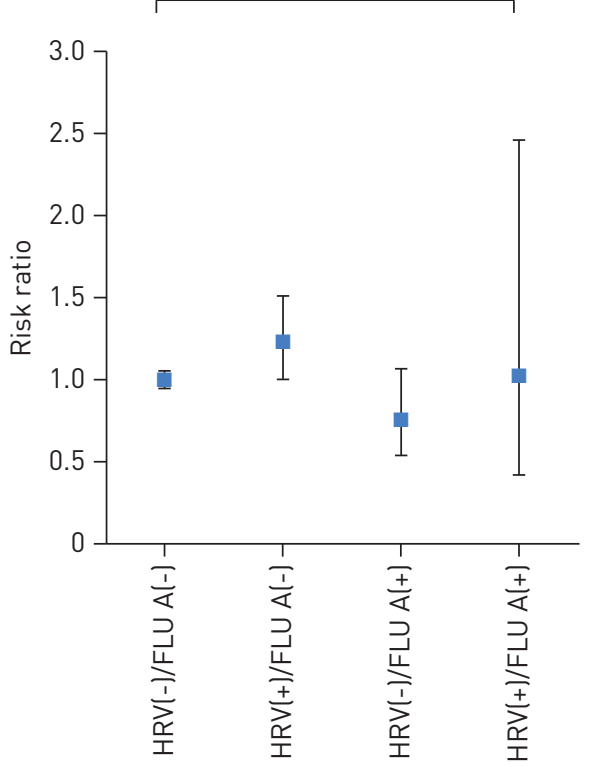

e)

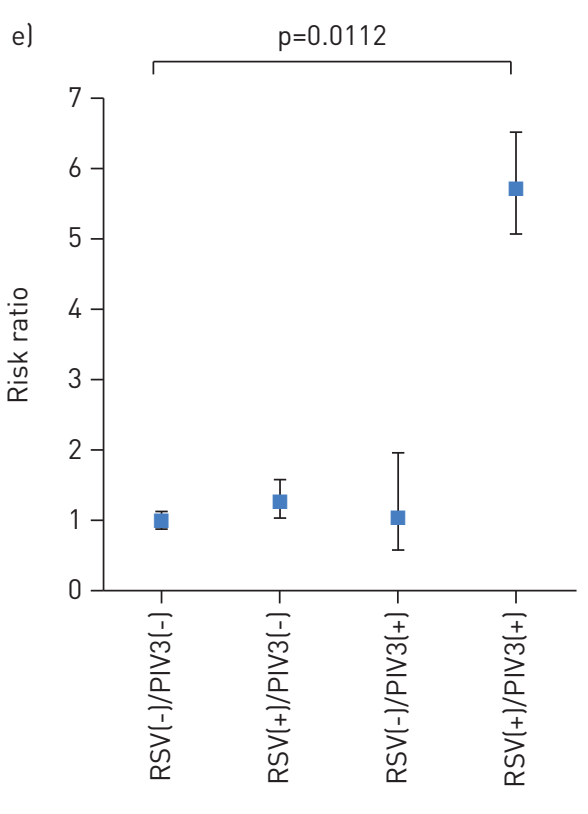

c)

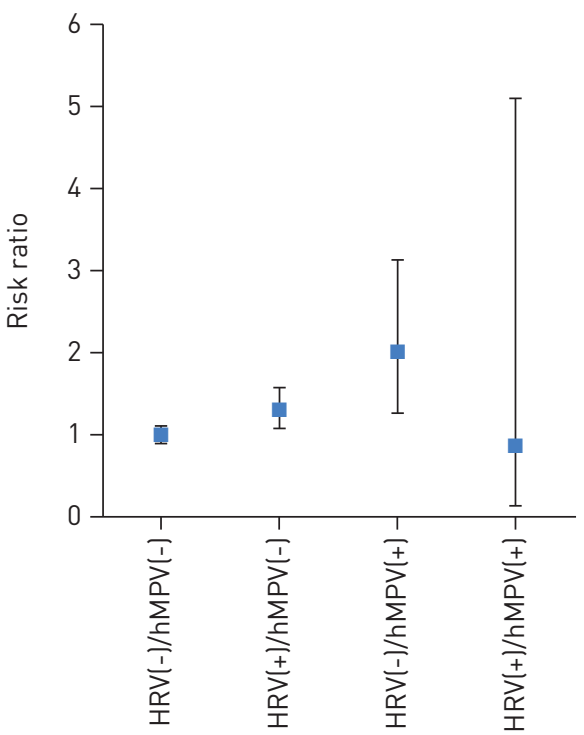

f)
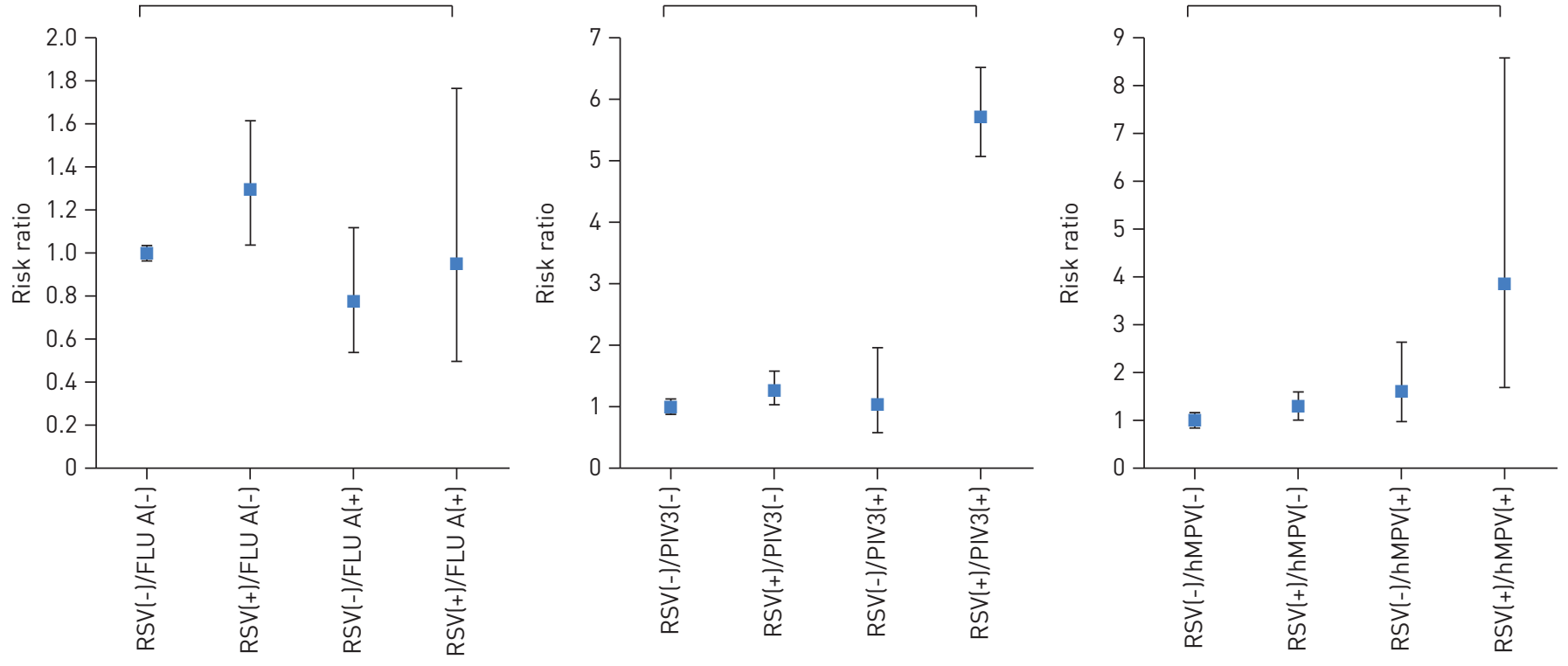

FIGURE 1 The effect of single-infection and co-infection with respiratory viruses on the risk of lower respiratory tract infections (LRTIs) among children aged $<5$ years in the Khanh Hoa Province, in Vietnam. Risk ratios were calculated for each category adjusting for other viruses. a) Human rhinovirus (HRV) and respiratory syncytial virus (RSV), adjusted for human metapneumovirus (hMPV). b) HRV and influenza A (FLU A), adjusted for RSV and hMPV. c) HRV and hMPV, adjusted for RSV. d) RSV and FLU A, adjusted for hRV and hMPV. e) RSV and parainfluenza virus (PIV)3, adjusted for HRV and hMPV. f) RSV and hMPV, adjusted for HRV. To evaluate trends of the effect of a single infection and co-infection on LRTIs, Chi-squared tests for trend were used, combining either single infection as one dummy category (i.e. $\mathrm{HRV}(+) / \mathrm{RSV}(-)$ and $\mathrm{HRV}(-) / \mathrm{RSV}(+)$ were regarded as one category) adjusting for other viruses.

\section{Respiratory virus co-infections and their interaction in the development of LRTIS}

Interestingly, our study found that co-infections of RSV with certain respiratory viruses increased the risk for LRTIs. Such effects appeared to depend on the combination of the different viruses; RSV with HRV, PIV3 or hMPV significantly increased the risk, while RSV with FLU A did not increase the risk. Recent studies have also shown that multiple viral co-infections are not rare in paediatric ARI cases [7, 14, 21], but these studies have not evaluated the effects of viral co-infection on the risk of LRTIs. Our observation may reflect the pathological effects of such synergistic interactions on childhood LRTIs.

Cytokines are thought to play an important role in the pathogenesis of RSV infection. It has been shown that RSV and FLU A stimulate the host immune system differently, especially in the context of the T-helper cell (Th)1 to Th2 balance. RSV infection has been shown to have an enhanced Th2 effect with increased 
production of interleukin (IL)-4, IL5 and a decrease in interferon (INF)- $\gamma$ levels; whereas a predominantly Th1 affect, with increased INF- $\gamma$ production, is observed in FLU A infection [22-24]. Therefore, coinfection of these two viruses might have neutralised their immunopathological effects in the respiratory tract, leading to this phenomenon. Future studies of the effects of viral co-infection on cytokines are necessary to clarify the interaction and underlying mechanism.

\section{Detection of HRV, RSV, hMPV or FLU A and risk of LRTIs}

Among children hospitalised for ARI, HRV, RSV and hMPV were associated with LRTIs, categorised by WHO IMCI guidelines, even after adjusting for the presence of other viruses. Although HRV infection was most commonly detected, the direct aetiological role of HRV in hospitalised ARI cases is questionable. Previous reports showed frequent detection of HRV in apparently healthy children [12]. Similarly, our casecontrol study demonstrated that one-third of healthy children were also positive for HRV and detection of HRV did not increase any risk for hospitalisation.

There could be several underlying reasons for why HRV was associated with LRTIs independent of the coinfection with other viruses, while there was a high HRV detection rate among healthy children. HRV loads [25] and serotypes [26] may play a role. ARI patients may have higher HRV loads than healthy controls, and/or may be infected with more pathogenic HRV while healthy control subjects harbour less pathogenic HRV. Other possibilities may be co-infection with other undetected respiratory viruses or bacteria. Further prospective studies to investigate the aforementioned possibilities are warranted to clarify the pathological role of HRV in ARI and LRTIs.

Interestingly, FLU A, one of the major causes of ARI hospitalisation, was not associated with the risk of LRTIs. This finding was consistent, even after analysing seasonal FLU A and H1N1 swine FLU A separately (data not shown). Lack of association of H1N1 swine FLU A with LRTIs was further surprising, since it has been associated with LRTIs or pneumonia more frequently than seasonal influenza worldwide [27, 28].

\section{Limitations of our study}

In this study we used multiplex PCR assays to detect 13 common respiratory viruses with high sensitivity. However, there is a possibility that some other viruses, which were not in our detection system, might be circulating in Nha Trang [29]. Another limitation was the lack of bacterial blood culture results in these cases. According to our previous study, there was a high rate of antibiotic usage before hospitalisation and thus blood culture-positive rates were very low $(<1 \%)$ [30]. Therefore, we did not conduct blood culture testing in this study.

This study used the modified WHO IMCI algorithms to define LRTIs instead of radiologically confirmed pneumonia as an outcome. Radiologically confirmed pneumonia has been used as an evaluation endpoint, especially for vaccine trials, as the definitions of the standardised radiological interpretation are very strict [31]. However, in this study we relied on the case definition of the WHO IMCI algorithms which are globally standardised and have been widely used, especially in the setting of developing countries.

In this study, severe LRTI cases were less likely to receive antibiotics before admission, were associated with wheezing and the paediatricians in Khanh Hoa General Hospital used steroids more frequently among them. Early treatment with antibiotics or medical attention or hospitalisation may reduce the risk of LRTIs in this study population. Our severe LRTI cases, defined by WHO IMCI algorithms, may have included many bronchiolitis cases. This may be the limitation of the WHO IMCI algorithms since it does not differentiate clearly between pneumonia and bronchiolitis cases.

\section{Conclusion}

RSV is the leading pathogen associated with the risk of ARI hospitalisation and LRTIs in central Vietnam. RSV played a major aetiological role either by itself or combination with other respiratory viruses. Thus, a vaccine or antiviral agents against RSV are urgently required to reduce severe paediatric ARI hospitalisation.

\section{Acknowledgments}

We thank the parents and participants in this study as well as staff from the Khanh Hoa Health Service (Nha Trang, Vietnam) and medical staff from Khanh Hoa General Hospital (Nha Trang, Vietnam) for their support in this study. We also thank staff from the Japan-Vietnam Friendship Laboratory at the National Institute of Hygiene and Epidemiology (Hanoi, Vietnam) and the Institute of Tropical Medicine (Nagasaki University, Nagasaki, Japan) for their kind support.

\section{References}

1 Black RE, Cousens S, Johnson HL, et al. Global, regional, and national causes of child mortality in 2008: a systematic analysis. Lancet 2010; 375: 1969-1987. 
2 Rudan I, Boschi-Pinto C, Biloglav Z, et al. Epidemiology and etiology of childhood pneumonia. Bull World Health Organ 2008; 86: 408-416.

3 O'Brien KL, Wolfson LJ, Watt JP, et al. Burden of disease caused by Streptococcus pneumoniae in children younger than 5 years: global estimates. Lancet 2009; 374: 893-902.

4 Watt JP, Wolfson LJ, O'Brien KL, et al. Burden of disease caused by Haemophilus influenzae type b in children younger than 5 years: global estimates. Lancet 2009; 374: 903-911.

5 Berkley JA, Munywoki P, Ngama M, et al. Viral etiology of severe pneumonia among Kenyan infants and children. JAMA 2010; 303: 2051-2057.

Hayden FG. Rhinovirus and the lower respiratory tract. Rev Med Virol 2004; 14: 17-31.

7 Williams JV, Edwards KM, Weinberg GA, et al. Population-based incidence of human metapneumovirus infection among hospitalized children. J Infect Dis 2010; 201: 1890-1898.

8 Weigl JA, Puppe W, Meyer CU, et al. Ten years' experience with year-round active surveillance of up to 19 respiratory pathogens in children. Eur J Pediatr 2007; 166: 957-966.

9 Dede A, Isaacs D, Torzillo PJ, et al. Respiratory syncytial virus infections in Central Australia. J Paediatr Child Health 2010; 46: 35-39.

10 Nair H, Nokes DJ, Gessner BD, et al. Global burden of acute lower respiratory infections due to respiratory syncytial virus in young children: a systematic review and meta-analysis. Lancet 2010; 375: 1545-1555.

11 Yoshida LM, Suzuki M, Yamamoto T, et al. Viral pathogens associated with acute respiratory infections in central vietnamese children. Pediatr Infect Dis J 2010; 29: 75-77.

12 Jartti T, Jartti L, Peltola V, et al. Identification of respiratory viruses in asymptomatic subjects: asymptomatic respiratory viral infections. Pediatr Infect Dis J 2008; 27: 1103-1107.

13 Bellau-Pujol S, Vabret A, Legrand L, et al. Development of three multiplex RT-PCR assays for the detection of 12 respiratory RNA viruses. J Virol Methods 2005; 126: 53-63.

14 Franz A, Adams O, Willems R, et al. Correlation of viral load of respiratory pathogens and co-infections with disease severity in children hospitalized for lower respiratory tract infection. J Clin Virol 2010; 48: 239-245.

15 Suzuki M, Thiem VD, Yanai H, et al. Association of environmental tobacco smoking exposure with an increased risk of hospital admissions for pneumonia in children under 5 years of age in Vietnam. Thorax 2009; 64: 484-489. Tulloch J. Integrated approach to child health in developing countries. Lancet 1999; 354: Suppl. 2, SII16-SII20.

17 McNutt LA, Wu C, Xue X, et al. Estimating the relative risk in cohort studies and clinical trials of common outcomes. Am J Epidemiol 2003; 157: 940-943.

18 Weber MW, Mulholland EK, Greenwood BM. Respiratory syncytial virus infection in tropical and developing countries. Trop Med Int Health 1998; 3: 268-280.

19 Hall CB, Weinberg GA, Iwane MK, et al. The burden of respiratory syncytial virus infection in young children. N Engl J Med 2009; 360: 588-598.

20 Sato M, Saito R, Sakai T, et al. Molecular epidemiology of respiratory syncytial virus infections among children with acute respiratory symptoms in a community over three seasons. J Clin Microbiol 2005; 43: 36-40.

21 Martin ET, Fairchok MP, Kuypers J, et al. Frequent and prolonged shedding of bocavirus in young children attending daycare. J Infect Dis 2010; 201: 1625-1632.

22 Bendelja K, Gagro A, Bace A, et al. Predominant type-2 response in infants with respiratory syncytial virus (RSV) infection demonstrated by cytokine flow cytometry. Clin Exp Immunol 2000; 121: 332-338.

23 Legg JP, Hussain IR, Warner JA, et al. Type 1 and type 2 cytokine imbalance in acute respiratory syncytial virus bronchiolitis. Am J Respir Crit Care Med 2003; 168: 633-639.

24 Aberle JH, Aberle SW, Pracher E, et al. Single versus dual respiratory virus infections in hospitalized infants: impact on clinical course of disease and interferon- $\gamma$ response. Pediatr Infect Dis J 2005; 24: 605-610.

25 Gerna G, Piralla A, Rovida F, et al. Correlation of rhinovirus load in the respiratory tract and clinical symptoms in hospitalized immunocompetent and immunocompromised patients. J Med Virol 2009; 81: 1498-1507.

26 Piralla A, Rovida F, Campanini G, et al. Clinical severity and molecular typing of human rhinovirus C strains during a fall outbreak affecting hospitalized patients. J Clin Virol 2009; 45: 311-317.

27 Chowell G, Bertozzi SM, Colchero MA, et al. Severe respiratory disease concurrent with the circulation of H1N1 influenza. N Engl J Med 2009; 361: 674-679.

28 Libster R, Coviello S, Cavalieri ML, et al. Pediatric hospitalizations due to influenza in 2010 in Argentina. N Engl J Med 2010; 363: 2472-2473.

29 Lau SK, Woo PC, Yip CC, et al. Coronavirus HKU1 and other coronavirus infections in Hong Kong. J Clin Microbiol 2006; 44: 2063-2071.

30 Anh DD, Kilgore PE, Slack MP, et al. Surveillance of pneumococcal-associated disease among hospitalized children in Khanh Hoa Province, Vietnam. Clin Infect Dis 2009; 48: Suppl. 2, S57-S64.

31 Cherian T, Mulholland EK, Carlin JB, et al. Standardized interpretation of paediatric chest radiographs for the diagnosis of pneumonia in epidemiological studies. Bull World Health Organ 2005; 83: 353-359. 